\title{
The link between ejection of a jet component and characteristics of variable continuum emission in the active galaxy $3 \mathrm{C} 390.3$
}

\author{
Tigran Arshakian*t \\ Max-Planck-Institut für Radioastronomie, Auf dem Hügel 69, 53121 Bonn, Germany \\ E-mail: tigar@mpifr-bonn.mpg.de
}

Tomaso Belloni

INAF-Brera Astronomical Observatory, Via E.Bianchi 46, I-23807, Merate, Italy

E-mail: tomaso.belloniabrera.inaf.it

\begin{abstract}
We study the correlations between the jet ejection event and changes in the continuum emission of the radio-loud galaxy 3C 390.3, using the archived monitoring data in radio, optical and X-ray. We present evidence for the link between the variable optical continuum and a stationary radio feature in the jet. The ejection of radio components happens during, or after, the dip in the X-ray light curve. Moreover, during the X-ray dip the flux variability is significantly reduced while the hardness ratio and its variance becomes harder. These findings strengthen the idea of similarity between active galactic nuclei (AGN) and microquasars, pointing towards a common physical mechanism acting in the disk-jet system. Other similarities are also discussed on the basis of comparision between 3C 390.3 and the microquasars GRS 1915+105 and Cyg X-1. If the analogy (based on linear mass scaling) between the ejection rates of the microquasar GRS 1915+105 and 3C 390.3 is correct, then the rate of ejections in 3 C 390.3 should vary between $\sim(0.01$ to 1$)$ ejections per year on a time scale of thousand years.
\end{abstract}

VI Microquasar Workshop: Microquasars and Beyond September 18-22, 2006

Como, Italy

\footnotetext{
*Speaker.

${ }^{\dagger}$ On leave from Byurakan Astrophysical Observatory, Aragatsotn province, 378433 Byurakan, Armenia
} 


\section{Introduction}

The AGN are the most powerful objects radiating over the entire electromagnetic spectrum. The bulk of continuum emission is thought to be produced by accretion of surrounding material onto the central massive nucleus (or black hole) which ejects it in the form of a relativistic collimated outflow (jet) of radio emitting plasma. On sub-pc-scales, the radio jet exhibits typically stationary and moving features of enhanced emission detectable with the Very Long Baseline Interferometry (VLBI) technique [1]. Moving components are ejected from the base of the jet very near to the central nucleus (or black hole). Stationary components of the jet can be associated with the internal oblique shocks, the emission and geometry of which depend strongly on the density distribution of the external medium [2]. The efficiency of coordinated radio VLBI and X-ray monitoring for investigating the disk-jet couplings was demonstrated by Marscher et al. [3]. They found that the dips in the X-ray continuum emission are associated with the epochs of ejection of radio blobs in the radio-loud galaxy $3 \mathrm{C} 120$ and interpreted this correlation as resulting from accretion of some part of the X-ray emitting disk into the black hole followed by ejection of a new radio component. Little is known about continuum emission variability and jet ejection coupling mainly because of the lack of coordinated multiwavelength monitoring campaigns in radio (VLBI) and other wavebands. To examine this coupling, we combine the jet ejection epochs in a radio-loud galaxy 3C 390.3 ( $z=0.00561)$ and archived monitoring data in radio, optical and X-ray (ROSAT and $R X T E$ ) bands. The dense $\mathrm{X}$-ray data allows zooming into the regions of $\mathrm{X}$-ray dips to study the $\mathrm{X}$-ray emission characteristics at the epoch of a jet ejection event.

\section{Correlations between the sub-pc-scale jet and variability of continuum emission in $3 \mathrm{C} 390.3$}

Ten epochs of Very Long Baseline Array (VLBA) imaging at $15 \mathrm{GHz}$ (obtained between 1994 to 2002 as a part of the $2 \mathrm{~cm}$ VLBA survey [1]) revealed the structure of the jet, the kinematics of jet components and the evolution of their emission on angular scales of $\sim 3$ milliarcseconds ( 1 mas=1.09 pc) [4]. Three stationary features (D, S1 and S2, see Fig. 1) and five moving components (C2-C6) with apparent speeds from 0.8 to $1.5 c$ were identified. The linear fits of moving components were used to estimate the epoch of their ejection from the D component (Fig. 2, red triangles), and the time at which they pass the stationary feature S1 (gray triangles). It was found that the bulk of variable radio emission ( $>90 \%$ on scales of two milliarcseconds) is radiated predominantly by the two stationary components $\mathrm{D}$ and $\mathrm{S} 1$ of the jet separated by $0.28 \pm 0.03$ mas $(\approx 0.3 \mathrm{pc}$ of projected distance).

\subsection{The link between radio and optical continuum emission}

In Fig. 2, the long-term variations of radio emission from D, S1 and the total radio emission (from D and S1) are superimposed onto optical continuum flux variations at $5100 \AA$. There is a significant correlation ( $>95 \%$ ) between variable radio emission from component $\mathrm{S} 1$ and optical continuum emission for a time delay of $\sim 0.2 \mathrm{yr}$ [4], while the total radio flux does not show any significant correlation with changes in the optical flux. This indicates that there is a physical link between variable optical continuum emission and variable radio emission from the stationary 


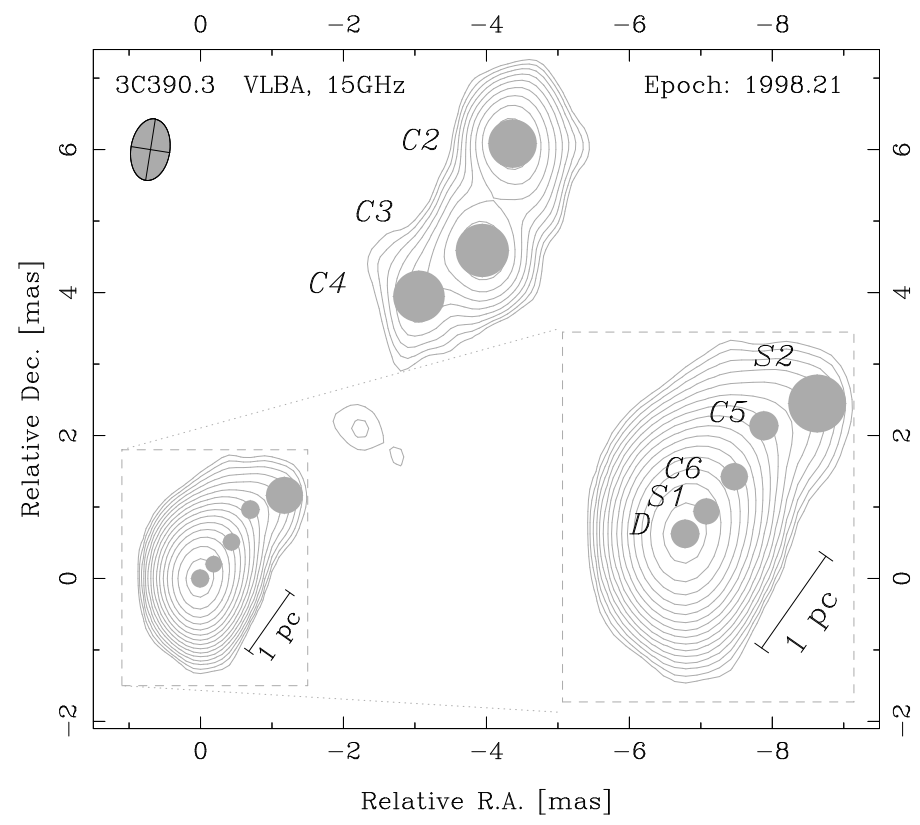

Figure 1: VLBA image of 3C 390.3 made in 1998.21 at $15 \mathrm{GHz}$ [4]. Model fit of the jet identified three stationary (D, S1, S2) and five moving components. The innermost two stationary components D and S1 are separated within 0.28 mas $\approx 0.3 \mathrm{pc}$ of projected distance. $\mathrm{D}$ is identified with the base of the jet and $\mathrm{S} 1$ is likely to be a standing shock of the jet

component $\mathrm{S} 1$ of the jet, suggesting that the bulk of optical continuum emission in the radio-loud galaxy 3C 390.3 is non-thermal and produced in the jet. Note that the variability amplitudes (see e.g. [5]) of the stationary components S1 and D of the jet are higher than those of the total radio, optical and X-ray continuum emission, with the variability amplitude of the S1 component being higher by a factor of $\geq 2$ (Fig. 3). This is a strong argument in favor of the fact that the source of variable continuum is localized in the innermost part of the jet and most likely to be associated with the S1 stationary feature of the jet.

\subsection{The link between the S1 component of the jet and optical continuum emission}

Further support for the link between S1 radio component and variable optical emission is provided by the correlation between the characteristic time (gray triangles in Fig. 2) at which the moving components $\mathrm{C} 4-\mathrm{C} 7$ pass the stationary feature $\mathrm{S} 1$. The epochs of passing of four $\mathrm{C} 4-\mathrm{C} 7$ components at $\mathrm{S} 1$ are delayed with respect to the maxima in the optical continuum (the average time delay is $0.18 \pm 0.06$ years). The null hypothesis that this happens by chance is rejected at a confidence level of $99.98 \%$ [4].

\subsection{The link between the $\mathrm{D}$ component and characteristics of the $\mathrm{X}$-ray emission}

The D component is likely to be the base of the jet, which is believed to be located near the central nucleus at a distance of $\geq 200$ Schwarzschild radii in or above a hot corona [6]. The ejection of plasma material from the base of the jet is thought to be coupled with the accretion of a part of disk material on the central nucleus. This scenario is supported by the correlation found between the dip in the X-ray flux associated with the accretion process and subsequent ejection of the jet 


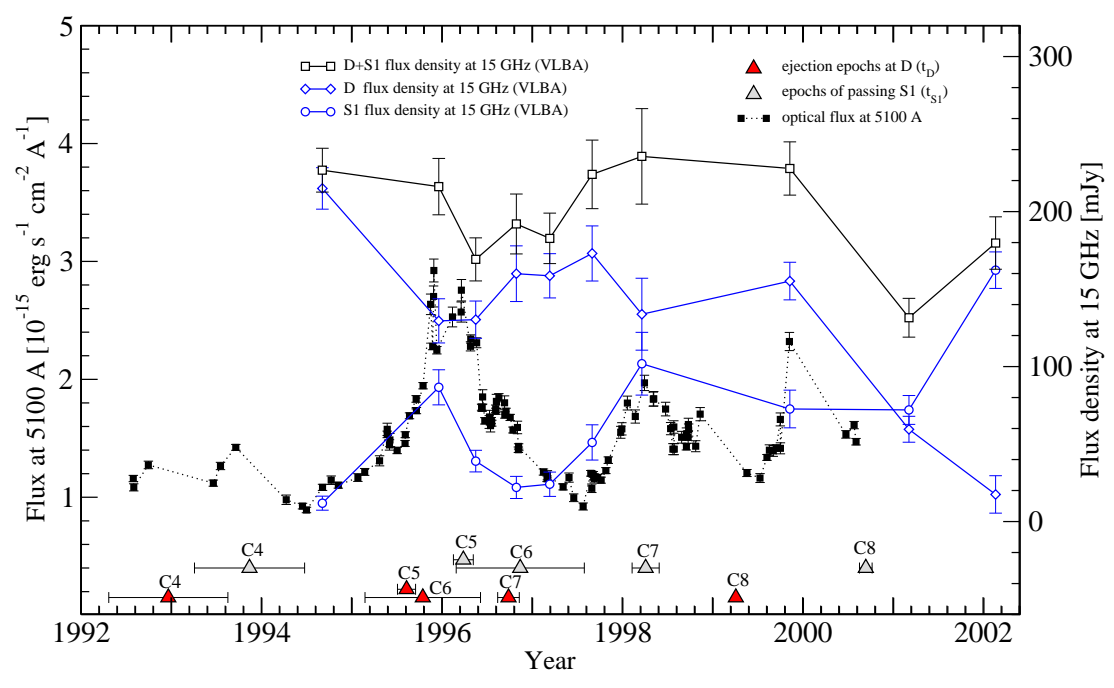

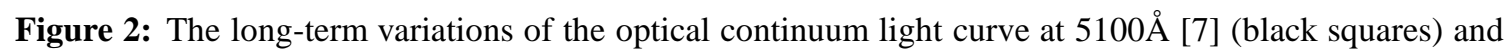
variations of radio flux density from the $\mathrm{D}, \mathrm{S} 1$ and $\mathrm{D}+\mathrm{S} 1$ components (empty blue diamonds, circle and empty black squares) are superimposed onto the times of ejection of radio components from the presumed base of the jet D (red triangles) and the times of their separation from the stationary component S1 (gray triangles). Adopted from [4]

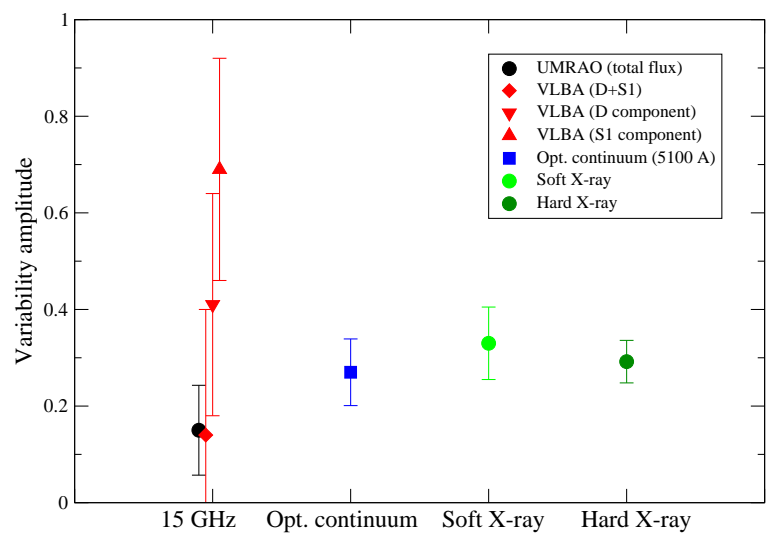

Figure 3: Amplitude of variability of continuum emission in 3C 390.3 at different frequencies: radio at 15 GHz (single dish total radio flux ${ }^{1}$, VLBA flux of D component, S1 and D+S1 components [4]), optical at $5100 \AA$ [7], soft X-ray at 0.1-2 keV [8] and hard X-ray at 2-20 keV [9]. 


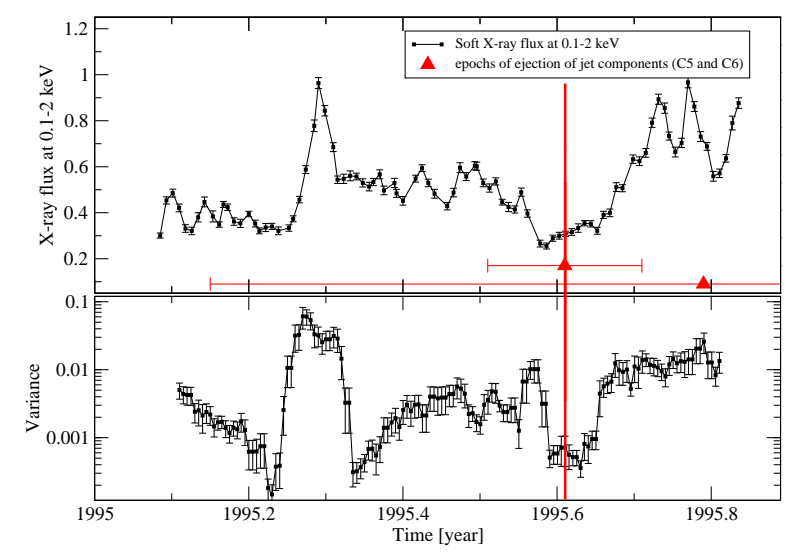

Figure 4: The epochs of ejection of the $\mathrm{C} 5$ and $\mathrm{C} 6$ radio components (red triangles) in 3C 390.3, time variations of the soft X-ray flux at $0.1-2 \mathrm{keV}$ (in units of Crab Nebula) and its variance taken over a sliding box with a width of 0.05 years.

component in the radio galaxy 3C 120 [3]. To check this correlation for 3C 390.3 and to explore new correlations, we used the archived X-ray monitoring data (the dense sampling of X-ray data allows the properties of the X-ray emission to be studied on time scales from 7-30 days).

The superposition of the ejection epochs of the C5 and C6 components and the soft X-ray light-curve (Fig. 4, upper panel) shows that the ejections occur during or after the dip in the X-ray flux. An interesting finding is that the variability of the X-ray flux becomes significantly lower during the dip in the X-ray flux (Fig. 4, lower panel) similar to what observed in the microquasar GRS $1915+105$. The average $X$-ray flux is higher by factor 1.5 after the radio ejection in $\sim 1995.6$, and there is a marginal evidence that the flux variability is also higher after the ejection of the C5 component. This may indicate that the total X-ray flux is enhanced by beamed variable X-ray emission of a new born relativistic radio component.

Leighly et al. [13] pointed out the remarkable feature of a single flare in 1995.3: the variance of the X-ray flux before and after the flare is reduced, evidencing that the X-ray variability is nonlinear. Another interesting feature is the increase of the flux after the flare by a factor of 2 (Fig. 4) which may indicate that the inner radius of the X-ray emitting disk shrinks after the flare. An interesting question is whether this isolated flare is a characteristic feature preceding the jet ejection.

The disk-jet coupling in 3C 390.3 is further evidenced from the hard X-ray monitoring data. In Fig. 5, the ejection time of the component $\mathrm{C} 8$ is compared with various characteristics of the $\mathrm{X}$-ray light-curve at 2-20 keV [9]. The jet ejection epoch coincides with the dips in the intensity of the X-ray flux and of the flux variability (left upper panel), the hardening of the spectrum (similar to $3 \mathrm{C} 120$ ) and a large variance of the hardness (left lower panel). Similar characteristics such as minimized flux variability, increased hardness and variance of the hardness are observed during the

\footnotetext{
${ }^{1}$ Data are taken from the University of Michigan Radio Astronomy Observatory online database (H.D. Aller and M.F. Aller, private communication).
} 

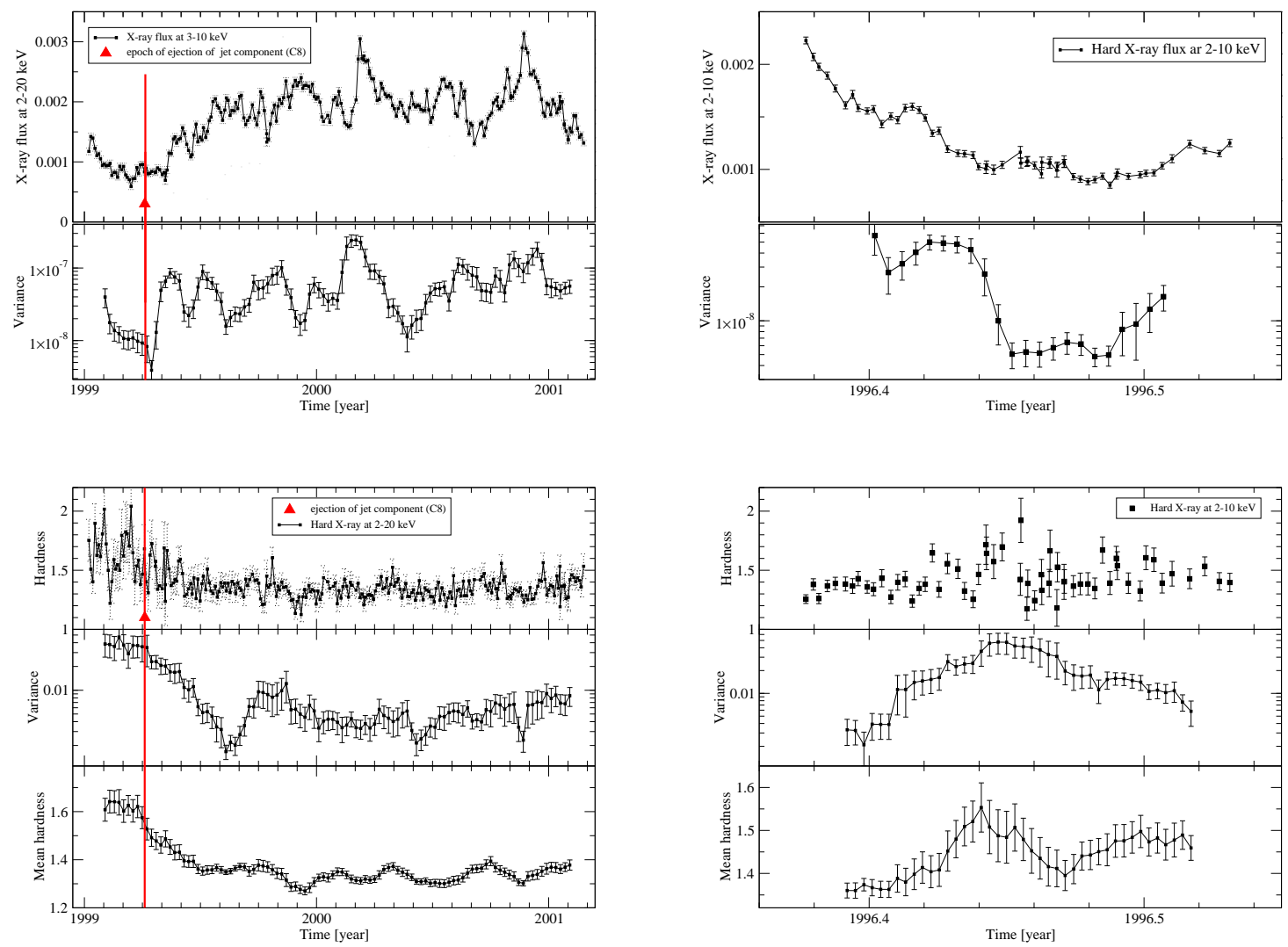

Figure 5: Left panels. The epoch of ejection of the component C8 (red vertical line) superimposed onto the changes of the RXTE light curve (at 2-20 keV [9]) and its variance (top panel), and the hardness ratio (defined as (15-6) keV/(6-2) keV), its variance and mean value (bottom panel) for a time period of about two years. Right panels. The daily changes of the RXTE light curve (at 2-10 keV [12]) and its variance (top panel), and hardness ratio ((10-6) $\mathrm{keV} /(6-2) \mathrm{keV})$, its variance and mean value (bottom panel) during a period of nearly two months. Note that component C7 was ejected in 1996.64 (see Fig. 2), approximately two months after the minimum in the $\mathrm{X}$-ray light curve and is not included here.

dip in the X-ray light-curve at 2-10 keV (Fig. 5, right panels).

Similar to 3C 120, the X-ray dip and the hardening of the spectrum are followed by the ejection of a radio component in the $3 \mathrm{C} 390.3$. This is a clear evidence that this correlation is a characteristic feature for radio-loud galaxies (see also [10] in this proceedings). In addition, we showed that during the X-ray dips the variance of the hardness increases while the variance of flux variability sharply becomes lower (Figs. 4,5), similar to what happens for the microquasar GRS 1915+105 [11]. This is a strong evidence for a common physical mechanism of disk-accretion and jet production processes in microquasars [14] and AGN.

\section{Similarities and differences with microquasars}

AGN and microquasars share similar activities displaying superluminal ejections of radio 
blobs from the central nucleus. The difference is in the energy output and mass, suggesting that microquasars are scaled-down versions of AGN. The scaling factor between 3C 390.3 and a typical microquasar is $\sim 10^{7}$ assuming a linear scaling between the central $\mathrm{BHs}\left(M_{\mathrm{BH}} \approx 3 \times 10^{8} M_{\odot}\right.$ for 3C 390.3 and 10-15 $M_{\odot}$ for GRS 1915+105). The X-ray light-curves of AGN harboring a supermassive black holes can be used to interpret small time scale variations occurring in microquasars, which are not achievable by present observations. The duration of the X-ray dip is about one to three months (see Figs. 4 and 5, left panel) which translates to $\sim(0.3$ to 1$)$ seconds for a microquasar. Another useful parameter is the jet ejection rate ranging from months to years in AGN [1]. The rate of ejections in 3C 390.3 is about one component in two years (Fig. 2) which scales to a one ejection in every six seconds in a microquasar. The high ejection rate of radio blobs in microquasars is associated with $\mathrm{X}$-ray dips occurring in the repeatable transitions between the hard and soft states [14]. In GRS 1915+105 the dips can take place on time scales from 1-2 seconds (see [15] in this proceedings) to 200 seconds in the $\mathrm{X}$ rays [14]. The high dip rate (one dip every two seconds) is comparable with the rate of expected ejections deduced from the mass scaling. The low dip rate in GRS 1915+105 translates to one ejection in about hundred years in 3C 390.3. If the analogy is correct, then the ejection rate in $3 \mathrm{C} 390.3$ should vary from $\sim(0.01$ to 1$)$ ejections per year on time scales of thousand years. We should bear in mind that the ejection of radio components in radio-loud AGN occurs always in the hard state. State transitions (hard to soft), at which the component is ejected in microquasars, have not been observed in AGN so far.

The 3C 390.3 shares also similar characteristics with the galactic black hole candidate Cyg X-1 in the low/hard state. Gliozzi et al. [9] argued that the simultaneous variations of spectral and intrinsic timing properties in 3C 390.3 are similar to variations observed in Cyg X-1 and other galactic black holes. In the same low/hard state, the ratio of timescales of the FWHM of X-ray flares in 3C 390.3 and Cyg X-1 is roughly consistent with the dynamical mass scaling [13] suggesting that the flare time scale is proportional to the mass of the black hole.

This research has made use of data obtained from the High Energy Astrophysics Science Archive Research Center (HEASARC), provided by NASA's Goddard Space Flight Center.

\section{References}

[1] K.I. Kellermann et al., Sub-Milliarcsecond Imaging of Quasars and Active Galactic Nuclei. III. Kinematics of Parsec-Scale Radio Jets (2004) ApJ 609539

[2] J.L. Gómez et al., Parsec-Scale Synchrotron Emission from Hydrodynamic Relativistic Jets in Active Galactic Nuclei (1995) ApJ 449 L19

[3] A.P. Marscher et al., Observational evidence for the accretion-disk origin for a radio jet in an active galaxy (2002) Nature 417625

[4] T.G. Arshakian et al., The source of variable optical emission is localized in the jet of the radio galaxy 3C 390.3, in proceedings of Relativistic Astrophysics and Cosmology - Einstein's Legacy meeting, November 7-11, 2005, Munich, Germany, edited by B. Aschenbach, V. Burwitz, G. Hasinger, and B. Leibundgut (2005) [astro-ph/0602016]

[5] P.M. Rodriguez-Pascual et al., Steps toward Determination of the Size and Structure of the Broad-Line Region in Active Galactic Nuclei. IX. Ultraviolet Observations of Fairall 9, (1997) ApJS 1109 
[6] A.C. Fabian, Broad Iron Lines in AGN and X-ray Binaries (2004) Ap\&SS 300197

[7] A.I. Shapovalova et al., Intermediate resolution $H \beta$ spectroscopy and photometric monitoring of $3 C$ 390.3. I. Further evidence of a nuclear accretion disk (2001) A\&A 376775

[8] K.M. Leighly et al., X-Ray Observations of the Broad-Line Radio Galaxy 3C 390.3 (2003) ApJ 483 767

[9] M. Gliozzi, I.E. Papadakis, C. Räth, Correlated spectral and temporal changes in 3C 390.3: a new link between AGN and Galactic black hole binaries? (2006) A\&A 449969

[10] A. Marscher, Relativistic Jets in AGN and their Relationship to the Central Engine, in proceedings of VI Microquasar Workshop: Microquasars and Beyond, PoS (MQW6) 025

[11] I.F. Mirabel et al., Accretion instabilities and jet formation in GRS 1915+105 (1998) A\&A 3309

[12] M. Gliozzi, R.M. Sambruna, M. Eracleous, Long-term monitoring of the broad-line galaxies 3 C 390.3 and 3C 120 with the ROSSI X-RAY TIMING EXPLORER (2003) ApJ 584176

[13] K.M. Leighly, P.T. O'Brien, Evidence for Nonlinear X-Ray Variability from the Broad-Line Radio Galaxy 3C 390.3 (1997) ApJ 48115

[14] R. Fender, T. Belloni, GRS 1915+105 and the Disc-Jet Coupling in Accreting Black Hole Systems (2004) ARA\&A 42317

[15] P. Soleri, T. Belloni, P. Casella, Fast aperiodic variability in the black hole binary GRS 1915+105: the timing signature of relativistic ejection events, in proceedings of VI Microquasar Workshop: Microquasars and Beyond, POS (MQW6) 043 